\title{
Silencing D. melanogaster lgr1 impairs transition from larval to pupal stage
}

\author{
Hans Peter Vandersmissen, Matthias Boris Van Hiel, Tom Van Loy, Rut Vleugels, Jozef Vanden Broeck* \\ KU Leuven, Animal Physiology and Neurobiology, Naamsestraat 59, PO Box 2465, Leuven, Belgium
}

\section{A R T I C L E I N F O}

\section{Article history:}

Available online 23 August 2014

\section{Keywords:}

Drosophila

Ecdysteroid

Glycoprotein hormone

Leucine-rich repeats containing GPCR (LGR)

Metamorphosis

Pupariation

\begin{abstract}
A B S T R A C T
G protein-coupled receptors (GPCRs) play key roles in a wide diversity of physiological processes and signalling pathways. The leucine-rich repeats containing GPCRs (LGRs) are a subfamily that is well-conserved throughout most metazoan phyla and have important regulatory roles in vertebrates. Here, we report on the critical role of Drosophila melanogaster LGR1, the fruit fly homologue of the vertebrate glycoprotein hormone receptors, in development as a factor involved in the regulation of pupariation. Transcript profiling revealed that lgr 1 transcripts are most abundant in third instar larvae and adult flies. The tissues displaying the highest transcript levels were the hindgut, the rectum and the salivary glands. Knockdown using RNA interference (RNAi) demonstrated that white pupa formation was severely suppressed in D. melanogaster lgr1 RNAi larvae. Associated with this developmental defect was a reduced ecdysteroid titer, which is in line with significantly reduced transcript levels detected for the Halloween genes shadow (sad) and spookier (spok) in the third instar lgr1 RNAi larvae compared to the control condition.
\end{abstract}

(c) 2014 Elsevier Inc. All rights reserved.

\section{Introduction}

Insects, which constitute over $80 \%$ of all animal species, have a huge impact on our environment and agriculture. A number of insects are infamous pest species or disease vectors, while others are very beneficial, for example as pollinators of flowering plants. Even though at first glance mammals and insects are very little alike, molecular genetic and physiological studies over the past years showed that fundamental aspects of many biological processes are well conserved throughout evolution.

In vertebrate physiology, the glycoprotein hormones are a group of key regulatory compounds. These heterodimeric proteins consist of an identical $\alpha$-subunit in combination with a hormonespecific $\beta$-subunit. Follicle stimulating hormone (FSH) is required in the maturation and maintenance of germ cells in both males and females, but it is also a determining factor for bone mass and for the growth of tumour blood vessels (Allan et al., 2010; Laan et al., 2012; Radu et al., 2010; Sun et al., 2006). Thyroid stimulating hormone (TSH) is produced in the pituitary gland and induces the growth of thyroid follicular cells and the release of

\footnotetext{
* Corresponding author. Fax: +32 16323902.

E-mail addresses: hanspeter.vandersmissen@bio.kuleuven.be (H.P. Vandersmissen), boris.vanhiel@bio.kuleuven.be (M.B. Van Hiel), tom.vanloy@bio.kuleuven.be (T. Van Loy), rut.vleugels@bio.kuleuven.be (R. Vleugels), jozef.vandenbroeck@bio. kuleuven.be (J. Vanden Broeck).
}

thyroid hormones which are important regulators of metabolism (Gogakos et al., 2010) and play a crucial role in the regulation of metamorphosis (Laudet, 2011). Luteinising hormone (LH) is responsible for the regulation of testosterone production in the male's Leydig cells (Chen et al., 2009). In females, it acts as the trigger for ovulation and is responsible for the formation of the corpus luteum which secretes progesterone. During pregnancy, the maintenance of the corpus luteum is taken over by human chorionic gonadotropin (hCG) which binds to the same receptor as LH and thus ensures the continued production of progesterone (Cole, 2010).

The receptors for the glycoprotein hormones are members of the leucine-rich repeats containing $G$ protein-coupled receptors (LGRs). Like all rhodopsin-type GPCRs, these receptors have a membrane-spanning domain consisting of seven $\alpha$-helices but their characteristically large ectodomain is their hallmark feature. This ectodomain holds the leucine-rich repeats that assume a horseshoe-shaped conformation and contain residues that are essential for the receptor's specificity (Kajava et al., 1995; Smits et al., 2003). Furthermore, these receptors feature a hinge region linking the ectodomain to the transmembrane domain. The hinge region has long been largely uncharacterised but recently, its structure was determined for the FSH receptor and it was predicted to play a key role in the activation of this receptor (Jiang et al., 2012).

The LGRs appear to be remarkably well conserved, with LGRs having been identified in Placozoa, Cnidaria, Nematoda, Arthrop- 
oda, Mollusca, Hemichordata, Echinodermata and Chordata. The family of LGRs can be divided into three major types based on the number of leucine-rich repeats (LRRs) in the receptor's ectodomain, their hinge region and the presence or absence of an LDLa motif. Type A LGRs display 7-9 LRRs and are characterised by a long hinge region whereas LGRs of the type $B$ feature twice as many LRRs (16-18) and have a shorter hinge region (but still larger than in type C LGRs). The hallmark of the type C LGRs is the presence of one or more LDLa motifs. The number of LDLa motifs allows for a further division of type C LGRs into two subtypes: a C1 type containing only one LDLa, which is well-conserved throughout many phyla, and a C2 type containing multiple LDLa motifs and having a distinct hinge region. This latter type of LGRs is rarer but has been identified in echinoderms and mollusks, as well as in Pediculus humanis corporis. Interesting with regards to the origin of LGRs are eight putative LGRs that were found in Trichoplax and which resemble type-C LGRs, but lack the LDLa motif. It has been proposed that these receptors might represent an ancient form of the type-C LGRs, prior to the incorporation of the LDLa motif, but it cannot be ruled out that Trichoplax LGR has lost its LDLa motif during the course of evolution (Van Hiel et al., 2012).

Although for invertebrate LGRs little evidence has currently been gathered on their biological functions, the important roles of their mammalian homologues and their evolutionary conservation suggest that they may be essential in these animals as well. So far, the only invertebrate LGR that was characterised extensively is LGR2 from D. melanogaster. This is the receptor for the heterodimeric neurohormone bursicon, which induces hardening and tanning of the cuticle and pupal case (Loveall and Deitcher, 2010; Luo et al., 2005; Mendive et al., 2005). Additionally, the hormone also co-ordinates the maturation of the wings in newly eclosed adult flies (Kimura et al., 2004; Natzle et al., 2008; Peabody et al., 2008).

In this paper, we have studied the closest fruit fly homologue of the vertebrate glycoprotein hormone receptors, D. melanogaster LGR1 (CG7665). This receptor, which was cloned in 1997 (Hauser et al., 1997), can be activated by the heterodimer GPA2/GPB5 (Sudo et al., 2005). Both subunits are cystine knot proteins that show similarities with the glycoprotein hormone subunits $\alpha$ and $\beta$, respectively. GPA2 and GPB5 were identified in the fruit fly based on their homology with the subunits of thyrostimulin, a hormone that was discovered to activate the vertebrate $\mathrm{TSH}$ receptor (Nakabayashi et al., 2002). Recent in situ hybridisation data showed expression of GPA2 and GPB5 in the same cells of the third instar CNS and using a GPB5-Gal4 line, the $\beta$-subunit producing cells were localised in larval and adult fruit flies (Sellami et al., 2011). Moreover, this study also pointed at the possible role of GPA2/GPB5 as an antidiuretic factor, while ablation of neurons producing this LGR1-ligand resulted in a developmental defect that led to reduced numbers of adult flies. In line with this hypothesis, GPA2/GPB5 was recently shown to affect the transport of $\mathrm{Na}+$ and $\mathrm{K}+$ across the hindgut of the mosquito, Aedes aegypti (Paluzzi et al., 2014).

In the present paper, we report on transcript profiling studies that were performed for $D$. melanogaster $\lg 1$ and for the genes encoding the hormone subunits, gpa2 (CG17878) and gpb5 (CG40041). Moreover, by means of an RNA interference (RNAi) construct targeting $\lg r 1$, we identified a remarkable developmental phenotype in D. melanogaster.

\section{Materials and methods}

\subsection{Phylogenetic analysis}

Multiple sequence alignment was performed in MAFFT (L-INS-i method) (Katoh et al., 2005, 2002) and converted to MEGA 5.10
(Tamura et al., 2011) wherein genetic distances between sequences were calculated and both a Maximum-Likelihood and a Neighbour-Joining tree were constructed. The Maximum-Likelihood tree was created using the Jones-Taylor-Thornton amino acid substitution model. The reliability of the tree was estimated by 2000-fold bootstrap re-sampling. The Neighbour-Joining tree was constructed using the Poisson amino acid substitution model The reliability of this tree was estimated by 5000 -fold bootstrap re-sampling.

\subsection{Rearing D. melanogaster}

Flies were kept at $25^{\circ} \mathrm{C}$ on a $12 \mathrm{~h}: 12 \mathrm{~h}$ light:dark cycle and fed on a standard diet of corn meal-yeast-agar medium. In the case of wild type flies, CantonS was used. For RNAi experiments, we used Act5C-Gal4 ( $w$;Act5C-Gal4/Cy $\left.{ }^{O}, g f p\right)$ as a driver line and as a responder, UAS-lgr1RNAi (line 13566 from the Vienna Drosophila RNAi Center). Two types of control lines were incorporated in the study: parental controls are flies from the responder and driver lines, sibling controls are the offspring of the cross that carry the balancer chromosome.

\subsection{Staging and weighing of third instar larvae}

Flies were allowed to lay eggs on an apple juice-agar medium (4\% apple juice, $4 \%$ agar). After $24 \mathrm{~h}$, first instar larvae were screened for GFP fluorescence (offspring control larvae) and transferred to apple juice-agar plates (diameter $50 \mathrm{~mm}$ ) covered with yeast paste (25 larvae per plate to prevent crowding). Staging of the larvae was performed by assessing spiracle morphology per time interval of $2 \mathrm{~h}$. For body mass determination, pools of 5 (timed) third instar larvae were weighed on a Sartorius Genius balance $(d=0.01 \mathrm{mg})$.

\subsection{Ecdysteroid titer measurements (enzyme immunoassay)}

Carefully timed third instar larvae were collected in pools of 5 larvae per tube. Extraction of ecdysteroids was performed as described by Warren et al. (2006). Quantification of the ecdysteroid titer was performed using an enzyme-linked immunoassay as described in more detail by Marchal et al. (2011).

\subsection{Desiccation survival}

UAS-lgr1RNAi responder flies were crossed with a heat shock responsive driver line, $(w ; H S-G a l 4)$. In the resulting offspring, RNAi was induced by heat shocking the flies at $37^{\circ} \mathrm{C}$ for $1 \mathrm{~h}$. Male flies from both experimental and parental control conditions (driver and responder line) were heat shocked the day of adult eclosion. Four days later, they were heat shocked again to boost the RNAi and after a recovery period of $5 \mathrm{~h}$, the flies were transferred to empty $1.5 \mathrm{ml}$ tubes capped with a cotton ball. Mortality of the flies was checked every hour, starting from $18 \mathrm{~h}$ after the start of desiccation. To exclude that observed effects were related to deprivation of food rather than water, in a parallel setup, flies were isolated in vials containing no food but an agar soil as a source of water.

\subsection{Determining water loss}

Male flies were weighed individually on a Sartorius Genius balance $(d=0.01 \mathrm{mg})$ to determine wet weight and were then dried in an oven at $65^{\circ} \mathrm{C}$ for $24 \mathrm{~h}$. The dry mass was determined and subtracted from the wet mass to calculate the water content. This was normalised to the wet mass of the flies. Another batch of flies was subjected to $16 \mathrm{~h}$ desiccation prior to determining their water content. 
2.7. Total RNA extraction, reverse transcription and quantitative realtime PCR ( $q R T-P C R)$

For the tissue samples, wandering third instar larvae and 4 day old adult wild type D. melanogaster were dissected in sterile PBS (Sigma-Aldrich). In the case of adults, the flies were briefly washed in ethanol prior to dissection. Tissues from males and females were collected separately. For each sample, three biological replicates consisting of 30 tissues were collected and pooled in RNase-free tubes which were kept in liquid nitrogen during the course of the dissections. Homogenisation was performed using brief rotor-stator drilling of the sample. RNA was extracted by use of the RNeasy micro kit (following the manufacturer's guidelines; Qiagen), including an on-column DNase digestion using RNase-free DNase (Qiagen) to eliminate possible genomic DNA contamination.

The "whole body" samples ( 25 individuals) for the developmental series were collected from wild type D. melanogaster in MagNA Lyser Green Beads tubes (Roche) and snap-frozen in liquid nitrogen. These were homogenised by a MagNA Lyser unit (Roche) (at $6500 \mathrm{rpm}$ for $30 \mathrm{~s}$ ) in $600 \mu$ proprietary buffer RLT (RNeasy mini kit, Qiagen) containing beta-mercapto-ethanol. The extraction method was followed as stipulated in the RNeasy mini kit protocol, including an on-column DNase digestion using RNase-free DNase (Qiagen).

Reverse transcription of the RNA samples was performed using Superscript III (Invitrogen) and random hexamer primers (Invitrogen) according to the manufacturer's instructions. The samples were diluted tenfold with MilliQ ${ }^{\circledR}$ water prior to use as a template in qRT-PCR (StepOne, Applied Biosystems).

qRT-PCR reactions were performed in 96 well format with each well containing $10 \mu \mathrm{l}$ of Fast SybrGreen master mix, $1 \mu \mathrm{l}$ each of fwd and rev primer $(10 \mu \mathrm{M})$ and $3 \mu \mathrm{l} \mathrm{MilliQ}{ }^{\circledR}$ and $5 \mu \mathrm{l}$ of diluted cDNA sample (i.e. following instructions of the Fast SybrGreen reagents supplier, Applied Biosystems). The PCR conditions were: $95^{\circ} \mathrm{C}$ for $10 \mathrm{~min}, 40 \times\left(95^{\circ} \mathrm{C}\right.$ for $3 \mathrm{~s}, 60^{\circ} \mathrm{C}$ for $\left.30 \mathrm{~s}\right)$. Primer sequences (synthesised by Sigma-Aldrich) are available in Supplementary Table 1. All primers were validated for PCR efficiency and amplicon specificity by analysis of the standard curve and melting curve. Normalisation was performed by using an external reference sample and the two housekeeping genes rp49 (ribosomal protein 49) and $\operatorname{cg} 1913$ (alpha-tubulin at 84B) [selected from a group of ten using GeNorm (Vandesompele et al., 2002)] according to the $\Delta \Delta \mathrm{Ct}$ method.

\section{Results}

\subsection{Phylogenetic analysis of well-described LGR sequences}

A phylogenetic analysis was performed with a limited set of well-annotated LGRs from different animal phyla using Maximum-Likelihood (Fig. 1) and Neighbour Joining (Fig. 2) analysis. In both trees, the three main types of LGRs (A-, B-, C-type) are indeed situated in distinct clusters. A schematic representation of the overall structural organization of the LGR-types can be found in Supplementary Fig. 1A, along with the multiple sequence alignment used for the creation of the phylogenetic trees. Based on BLAST searches, it is also interesting to notice that some animal species have lost receptor- and ligand-encoding gene combinations: for instance, the genome sequences of the hymenopteran model organisms, Apis mellifera and Nasonia vitripennis, seem to lack LGR1-, GPA2- and GPB5-encoding genes. In other animal species, gene multiplications appear to have occurred, as observed in the vertebrate lineage for the A-type LGRs (FSH, LH, TSH receptors) and their corresponding glycoprotein hormone beta subunits.

\subsection{Developmental transcript profiling}

To determine lgr1 transcript levels, qRT-PCR was performed on wild type samples representing the various developmental stages of D. melanogaster (Fig. 3A). Expression of the gene commenced in the embryonic stage but the relative transcript levels were more pronounced in the larval stages, particularly during the third instar. In the pupal stage, the lgr1 transcript level gradually declined, but rose again at the last day of the pupal stage (pharate adult). In adult flies, lgr1 transcript levels were consistently higher in males compared to females.

In addition to the transcript profile of $\lg r 1$, we also studied the transcript levels of the genes encoding the subunits of the described LGR1 ligand, gpa2 and gpb5. For both genes, transcripts were detected throughout the entire course of development of the fruit fly (Fig. 3B and C). The general trend displayed by the transcript signal was also similar between gpa2 and gpb5, except for first instar stage, at which the gpa2 transcript level showed a distinct peak. This was less pronounced in the case of gpb5. In adult flies, transcript levels for both genes were significantly higher in males than in females for most of the examined time points.

\subsection{Anatomical transcript profiling in the wandering third instar and adult stage}

In order to gain insight in the distribution of the $\lg 1$ transcript in the wandering third instar and in the adult fly, samples from carefully dissected tissues of these developmental stages were analysed using qRT-PCR (Fig. 4A and B). In the third instar, the most elevated transcript level was detected in the hindgut sample. Also, the salivary glands and the Malpighian tubules displayed significant transcript abundance. In adults, high transcript levels were detected in the hindgut and the rectum. Interestingly, a sexual dimorphism emerged as the salivary glands from male adults featured a higher transcript signal than in females. Furthermore, lgr1 transcript abundance was significantly higher in testes than in ovaries. The signals from other reproductive tissues were not compared statistically given their different functional natures. However, it is clear that the transcript level in the seminal vesicle was much stronger than the signals detected in female reproductive tissues.

Analyses of the tissue distribution revealed that gpa2 and gpb5 transcripts displayed the highest levels in the central nervous system (CNS) of third instar larvae and adults (thoracic-abdominal ganglia) (Fig. 4C-F). Outside the CNS, gpb5 transcripts were distributed rather evenly throughout the various tissues of the larval body. Gpa2 transcripts were also detected in all larval tissue samples, but were significantly more abundant in the developing male gonad compared to the female gonad. In adults, testes displayed significantly higher transcript abundance for gpa2, whereas transcripts for gpb5 were more abundant in tissues of the female reproductive system.

\subsection{Lgr1 RNAi larvae fail to form white pupae}

In order to study developmental effects associated with LGR1 insufficiency, RNAi was induced using an Act5C-Gal4 driver line which resulted in a $50 \%$ decrease of transcript abundance in third instar larvae compared to sibling control larvae (Suppl. Fig 4A). Whereas control larvae underwent white pupa formation (WPF) approximately 2 days after having moulted to the third instar stage, only $22 \%( \pm 10 \%)$ of $\lg 1 \mathrm{RNAi}$ larvae proceeded with WPF (Fig. 5). If lgr1 RNAi larvae did undergo WPF, this happened with a 2-4 days delay in comparison to sibling control larvae. The lgr1 


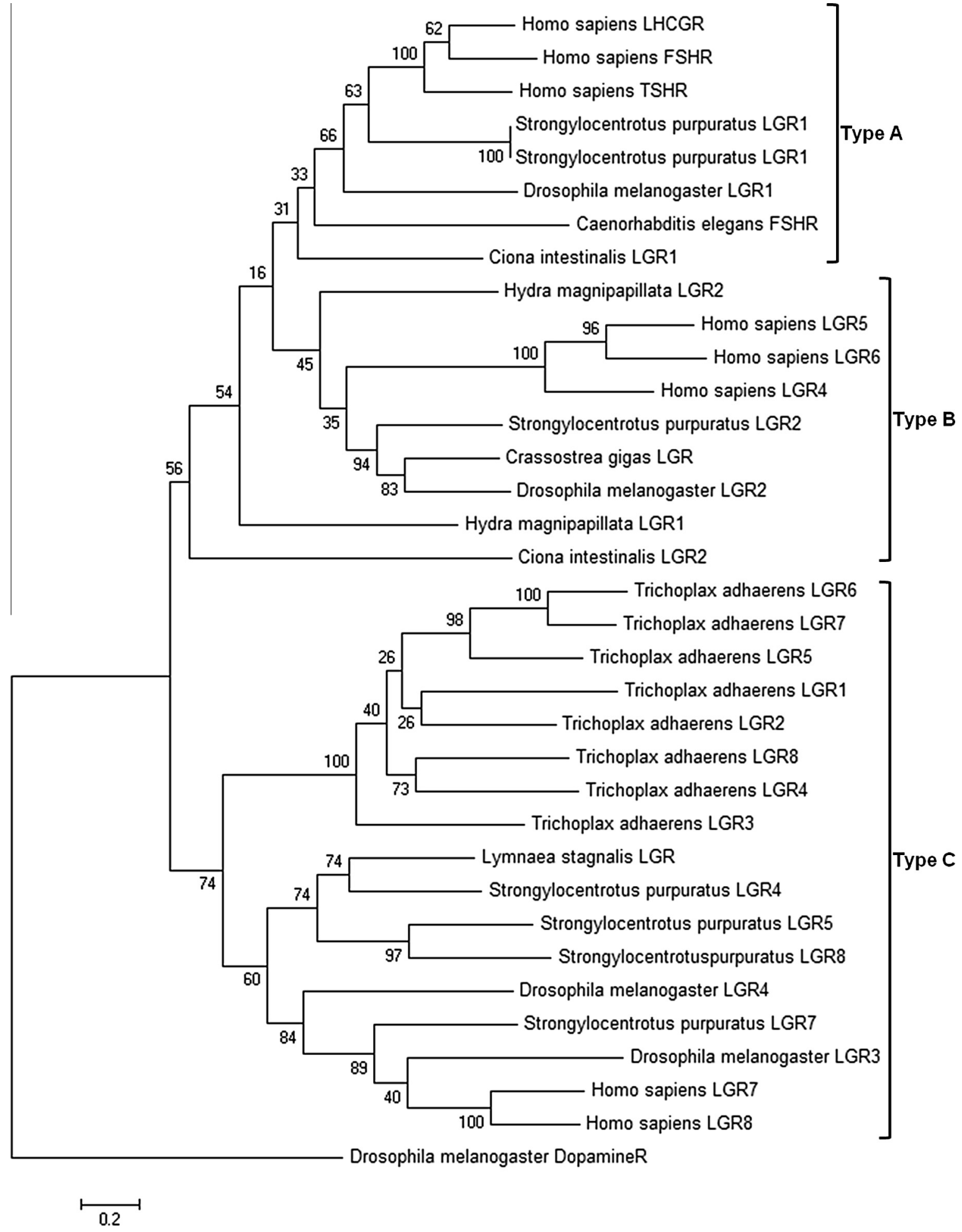

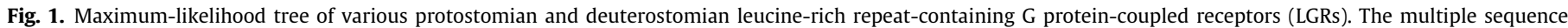
alignment is shown in Supplementary Fig. 1B.

RNAi pupae arrested development in diverse stages of metamorphosis, generally prior to reaching the pharate adult stage. Only in very rare cases ( $1 \%$ of pupae formed), adult flies eclosed from the pupal case. Larvae that failed to undergo WPF stayed alive as third instars until death occurred, up to 15-20 days after moulting to the third instar stage.
These larvae did not display full wandering behaviour, i.e. they had a tendency to leave the food medium as expected for wandering larvae but they always returned to the food. Given this observation, we have studied the transcript profile of sgs4 (salivary gland secretion 4) (Fig. 6A), since it is known that this is one of the genes of which the expression is associated with the switch to wandering 


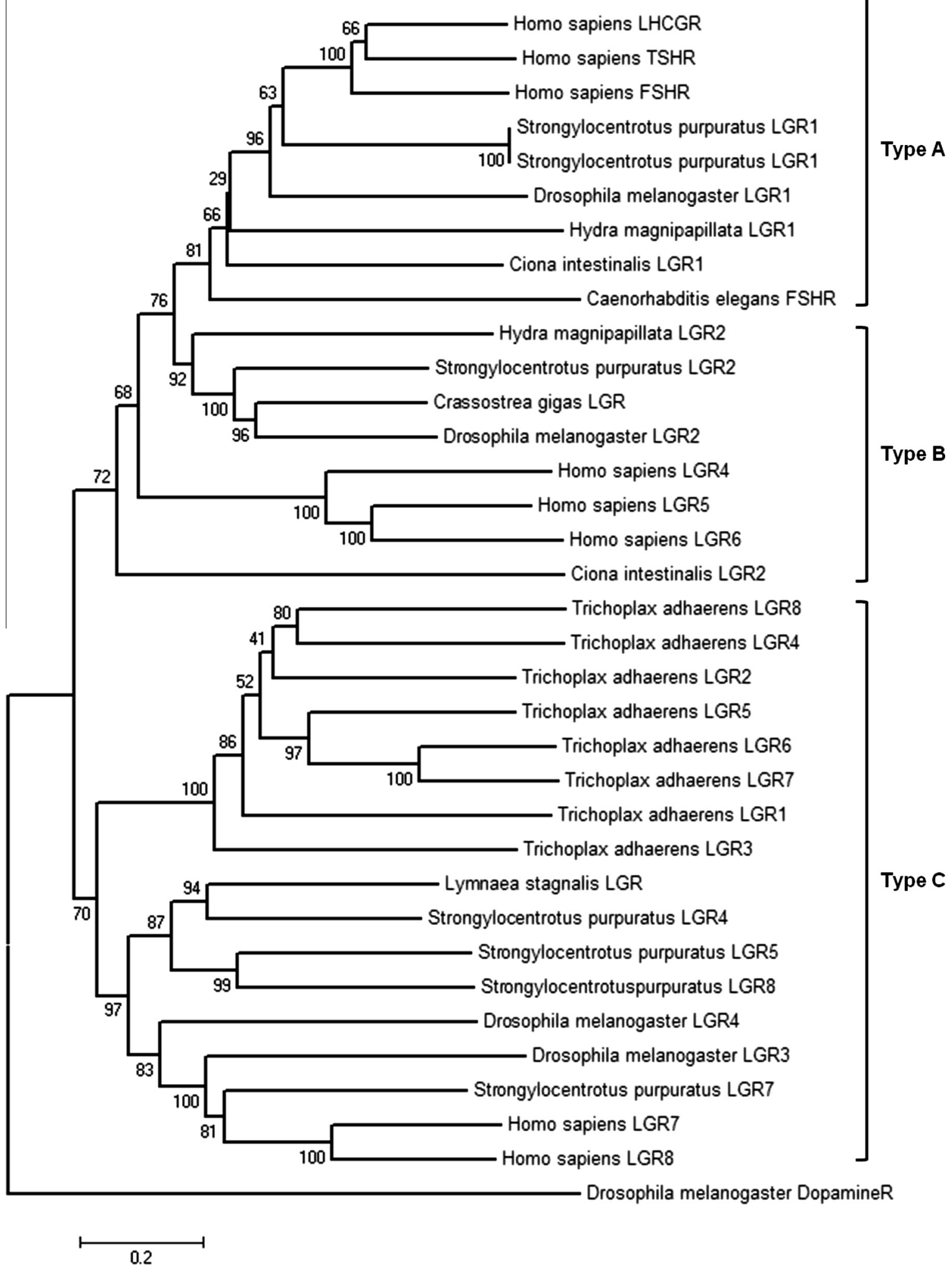

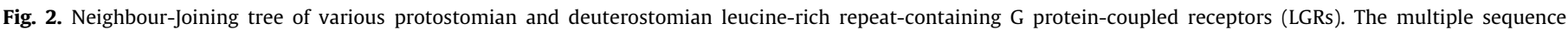
alignment is shown in Supplementary Fig. $1 \mathrm{~B}$.

behaviour. Using qRT-PCR, only basal sgs4 transcript levels were detected in lgr1 RNAi larvae throughout the third instar stage (Fig. 6A). In contrast, in the control larvae, sgs4 transcript was nota- bly present from $24 \mathrm{~h}$ after the third instar moult and levels peaked in the last $12 \mathrm{~h}$ of the wandering stage, leading up to WPF. For another gene of which the expression is upregulated in the wan- 

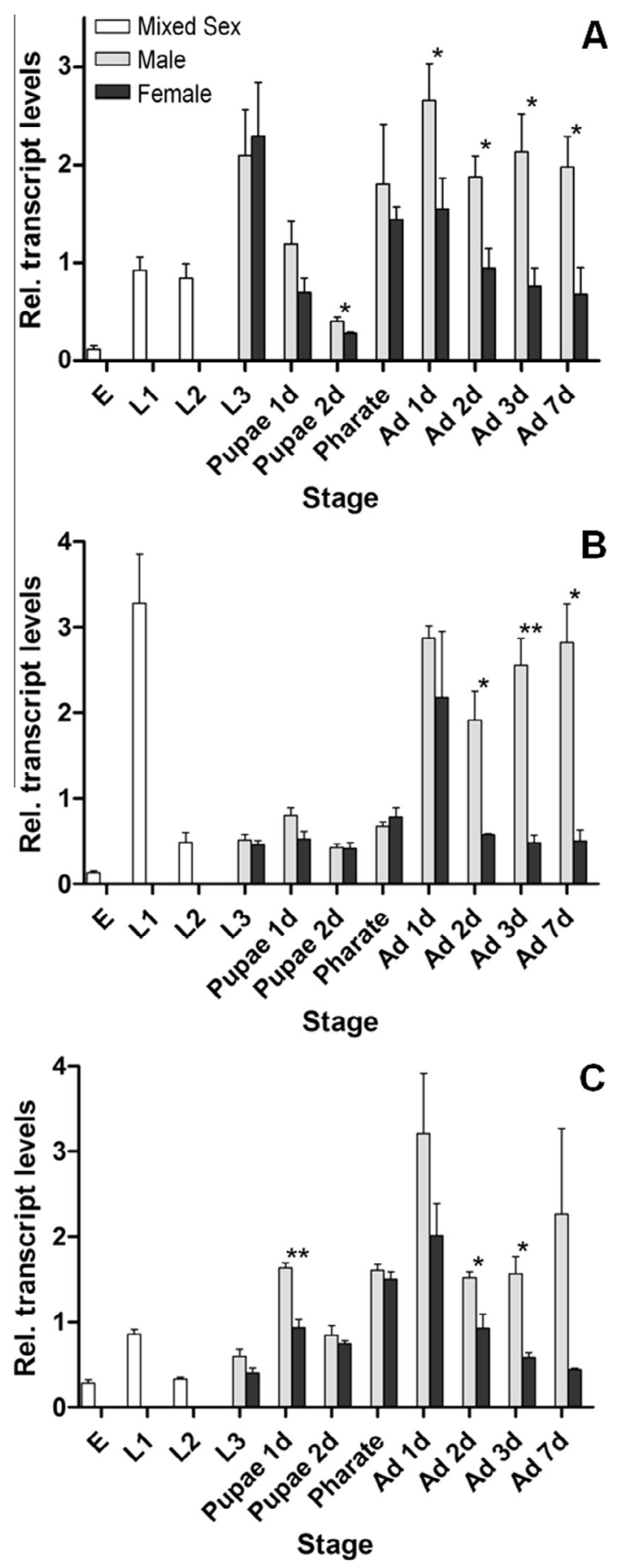

Fig. 3. Transcript profile of $\operatorname{Lgr} 1$ (A), Gpa2 (B) and Gpb5 (C) during the course of $D$. melanogaster development. Mean (and SEM) of 3 biological replicates (25 individuals). Significance of the gender difference was determined by $t$-test $\left({ }^{*} p<0.05\right.$ ${ }^{* *} p<0.01$ ). [E: embryo, L1: first larval instar, L2: second larval instar, L3: wandering third larval instar, Pupae 1d: first day of pupal stage, Pupae 2d: second-third day of pupal stage, Pharate: final day of pupal stage, Ad xd: adult $\mathrm{x}$ days post eclosion].

dering stage, ilp6, transcript levels rose steeply in the control larvae as they progressed in the third instar stage. In contrast, ilp6 transcript levels were significantly lower in lgr1 RNAi larvae (Fig. 6B).

\subsection{Lgr1 RNAi larvae do not gain sufficient body mass}

As the accumulation of body mass is important for the larva to reach the critical weight threshold and thus, to pupariate, the body mass of control and lgr1 RNAi larvae was determined during the third instar stage (Supplementary Fig. 2). At the time of the third instar moult, the body masses of lgr1 RNAi and control larvae were not significantly different. Control larvae nearly doubled their body mass during the first $24 \mathrm{~h}$ after the third instar moult, after which their mass slightly decreased during the wandering stage (24-48 h after moulting). In contrast to controls, lgr1 RNAi larvae gained little body mass during the first $24 \mathrm{~h}$, after which their mass did not change significantly.

\subsection{Lgr1 RNAi larvae fail to accumulate high ecdysteroid titers}

Since it is well established that ecdysteroid titers peak around the time of WPF, we monitored the ecdysteroid titers in whole larvae at different time points during the third instar stage. In parental and sibling control conditions, the ecdysteroid titer was low during most of the third instar stage and increased precipitously around the time of WPF. In contrast, the ecdysteroid titers in lgr1 RNAi larvae were low throughout the entire prolonged third instar stage. In the case of lgr1 RNAi larvae that did pupariate, the ecdysteroid titer was more elevated, although it seemed to take longer for these larvae than for the controls to reach the required threshold (Fig. 7A). In order to attempt rescuing the observed defects, lgr1 RNAi larvae were fed on a medium containing 20-hydroxyecdysone (20E) (Suppl. Fig. 4B). The animals treated in this way indeed formed pupae but still did not develop into adults.

To explore the origin of the low ecdysteroid titers in lgr1 RNAi larvae, a qRT-PCR study was performed on closely timed third instars to monitor the transcript levels of genes associated with ecdysteroid biosynthesis. Only the transcript levels of spookier (spok) and shadow (sad) were significantly reduced in lgr1 RNAi larvae (Fig. 7B and C). Sad encodes a mitochondrial cytochrome P450 that mediates the conversions of 2-deoxyecdysone to ecdysone and of ketotriol (2,22-dideoxyecdysone) to 22-deoxyecdysone (Warren et al., 2002). The exact function of SPOK remains unknown and this enzyme likely has a role in the 'black box', the uncharacterised part of the ecdysone biosynthesis pathway (Ono et al., 2006, 2012).

No significant differences could be observed in the transcript levels of the Halloween genes disembodied (dib), shade (shd), phantom (phm) or spook (spo). Also, the transcript levels for prothoracicotropic hormone ( $p t t h)$, neverland ( $n v d$ ) and moulting deficient ( $m l d$ ) (Supplementary Fig. 3) were not significantly altered in lgr1 RNAi larvae in comparison to sibling controls. These latter are genes that are associated with the regulation of ecdysteroid biosynthesis, but are not members of the Halloween gene family.

To determine whether the effect of LGR1 signalling on development was located in the prothoracic glands (PG), several Gal4 driver lines were used to silence lgr1 in the PG (part of the ring gland) specifically. However, none of these driver lines could elicit the previously described developmental phenotype (results not shown).

\subsection{Knockdown of lgr1 expression lowers desiccation tolerance}

The most pronounced levels of $\lg 1$ transcript were found in the hindgut of larval and adult flies and this organ is responsible for the reuptake of water from the faeces. Based on these observations, a possible role for LGR1 in the regulation of water reuptake was hypothesised. Defects in this process would likely lead to a more rapid death when facing desiccation. Since general induction of lgr1 silencing did not yield a sufficient number of adults to perform these assays, RNAi was induced by heat shock early in the adult stage. Survival curves of male adults kept deprived of a water or food source indicated that lgr1 RNAi flies showed a significantly reduced survival in these conditions com- 

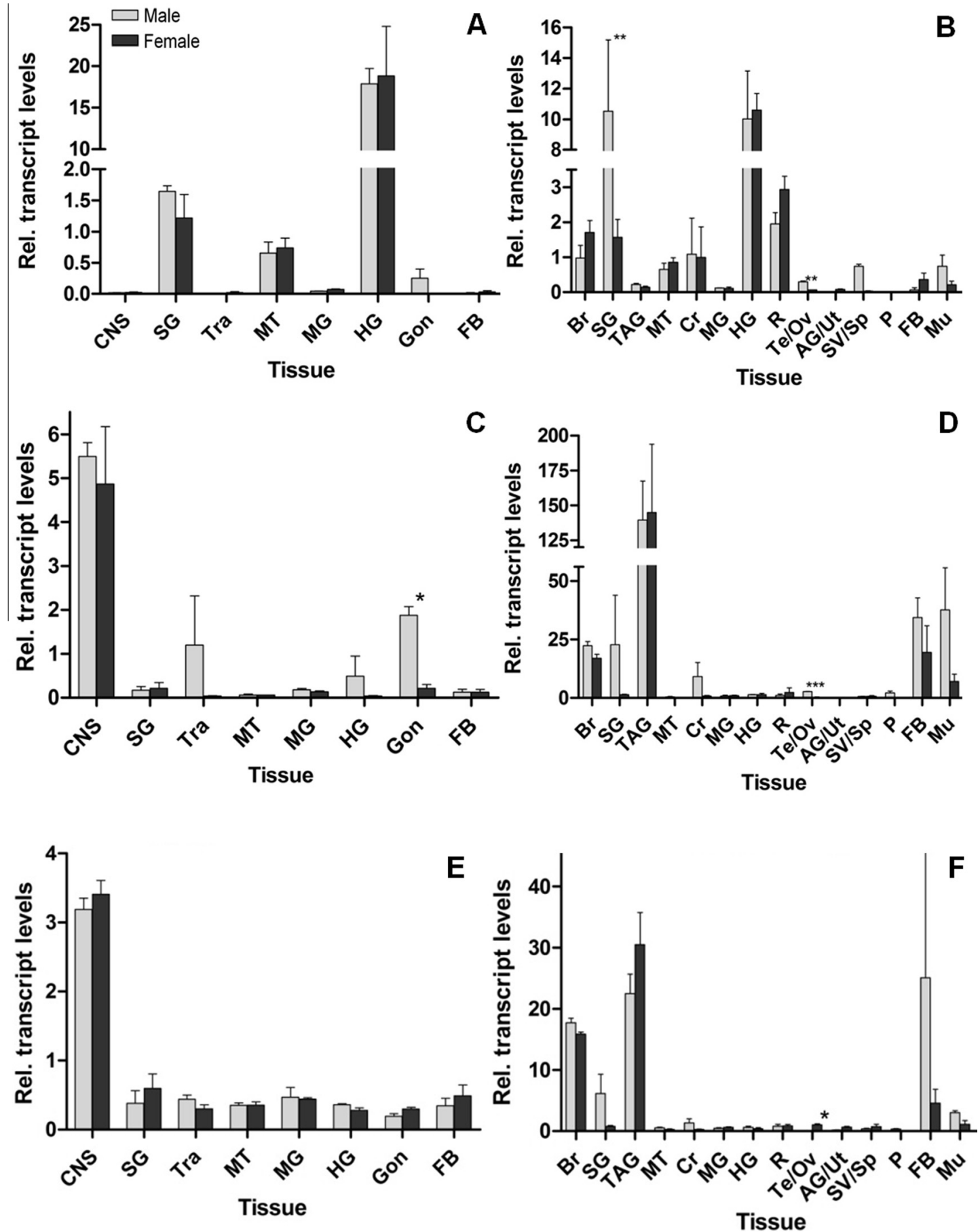

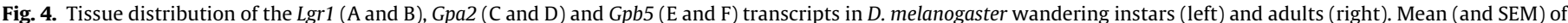

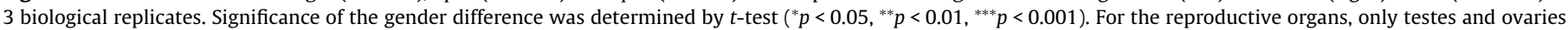

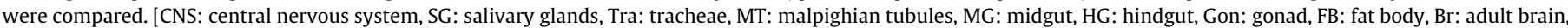

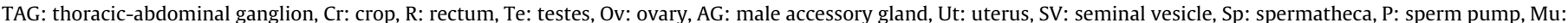
muscle].

pared to parental control lines (Log Rank Test) (Fig. 8A). This was not the case when flies were kept in the absence of food, but with access to water (data not shown). In addition, the water content of heat shock induced lgr1 RNAi flies was significantly lower compared to control flies. Also, after a period of desiccation of $16 \mathrm{~h}$ the control had not lost a significant amount of water whereas lgr1 RNAi flies did (Fig. 8B).

\section{Discussion}

\subsection{Phylogenetic analysis of well-described LGR sequences}

The occurrence of LGRs from Cnidaria and Nematoda within the A-type cluster, to which D. melanogaster LGR1 belongs as well, may suggest that this LGR-type is the more ancient one (Fig. 1 and Fig. 2). However, relatively frequent gene losses or gene multiplica- 


\section{Developmental phenotype for LGR1}

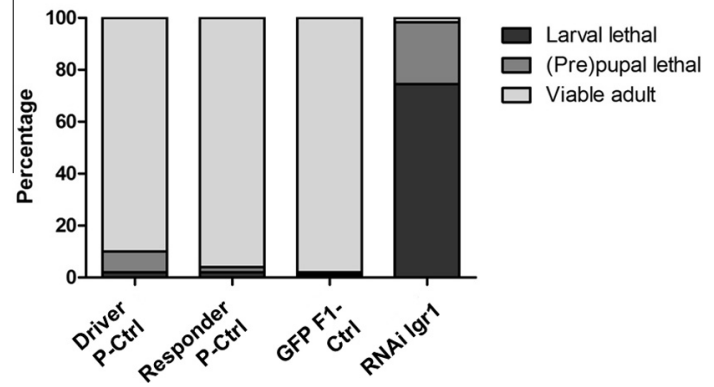

Fig. 5. Developmental phenotype associated with $\lg 1$ silencing $(n=250-300)$. Pctrl: parental control flies - Driver: Act5C > Gal4, Responder: UAS-lgr1 RNAi; GFP F1-Ctrl: offspring of the (driver $\times$ responder) cross carrying no Gal4-driver sequence (no induced RNAi effect); RNAi lgr1: offspring carrying both Act5C > Gal4-driver and UAS-lgr1 RNAi responder sequences (RNAi occurring).
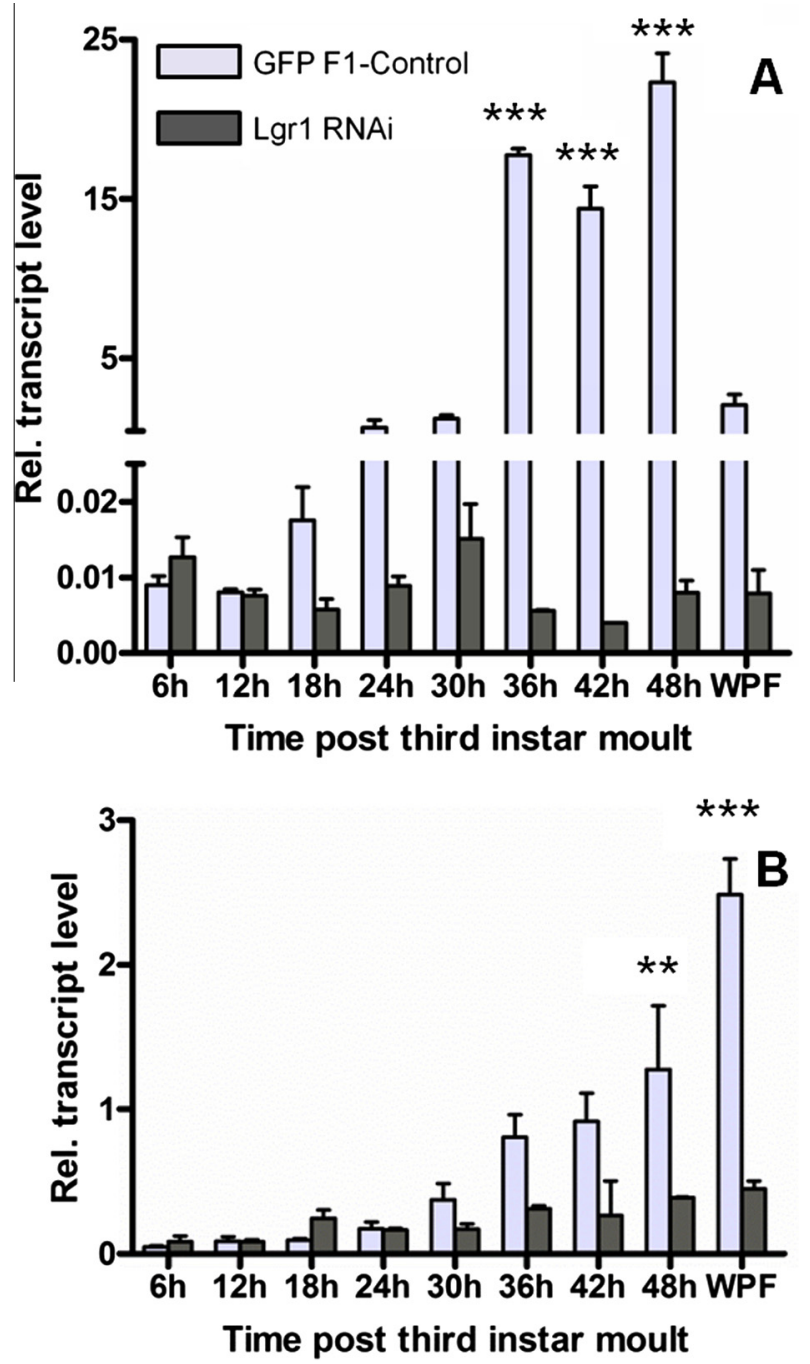

Fig. 6. Relative transcript levels of $\operatorname{sgs}_{4}$ (A) and ilp6 (B) during the third instar stage. Mean (and SEM) of 3 biological replicates. Significance of the difference was determined by Two way ANOVA with Bonferroni post hoc test $\left({ }^{* *} p<0.01\right.$, $\left.{ }^{* * *} p<0.001\right)$.

tions can be observed in different metazoan evolutionary lineages. An interesting example of LGR gene duplication in insects are the two type A LGRs that are encoded in Tribolium castaneum, making this beetle the only invertebrate so far to have multiple LGR type A receptors. Indeed whereas generally only one type A LGR can be distinguished in an organism's genome, vertebrate species feature 3 of these receptors. Gene loss in the LGR family is well illustrated by the nematode in which only a type A LGR can be found. While in many model organism genomes one can speculate that missing LGRs are still waiting to be annotated, the genome of Caenorhabditis elegans has been studied so extensively that the loss of B or C type LGRs can be considered a fact.

Given the ancient origin of this N-terminal ectodomain containing GPCR subfamily, it is possible that the genomic events situated at its very early evolutionary origin, which may have involved the fusion of an LRR-containing domain with a serpentine (7TM) domain, cannot be readily reconstructed anymore. Given the notable degree of conservation between LGRs throughout different animal phyla and the essential functions that the currently described LGRs fulfil, we propose that these receptors may in general take up roles that are key to development, reproduction and general physiology. Although much remains to be uncovered on the roles that LGRs take up in organisms outside the mammalian lineage, our previous work on the fruit fly LGR2 receptor, identifying it as the receptor for the neurohormone bursicon (Mendive et al., 2005; Luo et al., 2005), as well as this current study on LGR1 support this hypothesis.

\subsection{Transcript profiling of lgr1, gpa2 and gpb5}

Our analyses of the transcript levels for $\lg r 1$ in $D$. melanogaster clearly indicated that the gene is already active in the embryonic stage. Therefore, lgr1 may be involved in a developmental process. Transcript could be detected throughout the entire life cycle of the fruit fly. Previous studies already clarified that the transcription of $\lg 1$ in the fruit fly embryo likely commences around 8-16 h after egg deposition (Hauser et al., 1997). Our data further indicated that the transcript level was significantly higher in the larval stages compared to the embryo. Furthermore, the lgr1 mRNA level in the wandering third larval stage was more prominent than in the other larval stages. During metamorphosis, the relative transcript level of lgr1 decreased, which is in line with the receptor being important in the transition to the pupal stage, as suggested by the results of our RNAi study. In the pharate adult stage, the transcript level rose again, which may point to LGR1 being a regulator at this point in development as well. In the male adult, the relative lgr1 mRNA quantity remained high, but in the female, this level decreased after the first day of adulthood. These findings are supported by other studies which previously established that lgr1 mRNA was more abundant in males than in females (Hauser et al., 1997) and current RNA sequencing data support the developmental transcript profile determined in our experiments (Graveley et al., 2011).

A ligand for LGR1 is the heterodimer composed of GPA2 and GPB5, both of which are related to the glycoprotein hormone subunits (Sudo et al., 2005). Therefore, it is plausible that the transcript profiles for both genes would display similarities. Especially from the third instar onwards, the trends in the transcript profiles were indeed alike for these genes. An exception was the precipitous peak in transcript abundance for gpa2 in the first instar stage, which was less pronounced in the case of gpb5. In adult flies, the transcript levels for both gpa2 and gpb5 were significantly lower in females compared to males, which was also described for $\lg 1$. In general, there were similarities between the transcript profiles of gpa2, gpb5 and lgr1, which is in line with GPA2/GPB5 being a ligand of LGR1. Also, there is a general correspondence between our results and modENCODE RNA sequencing data (Graveley et al., 2011).

With regard to tissue distribution, the highest $\lg 1$ transcript levels were detected in the hindgut, the Malpighian tubules and 

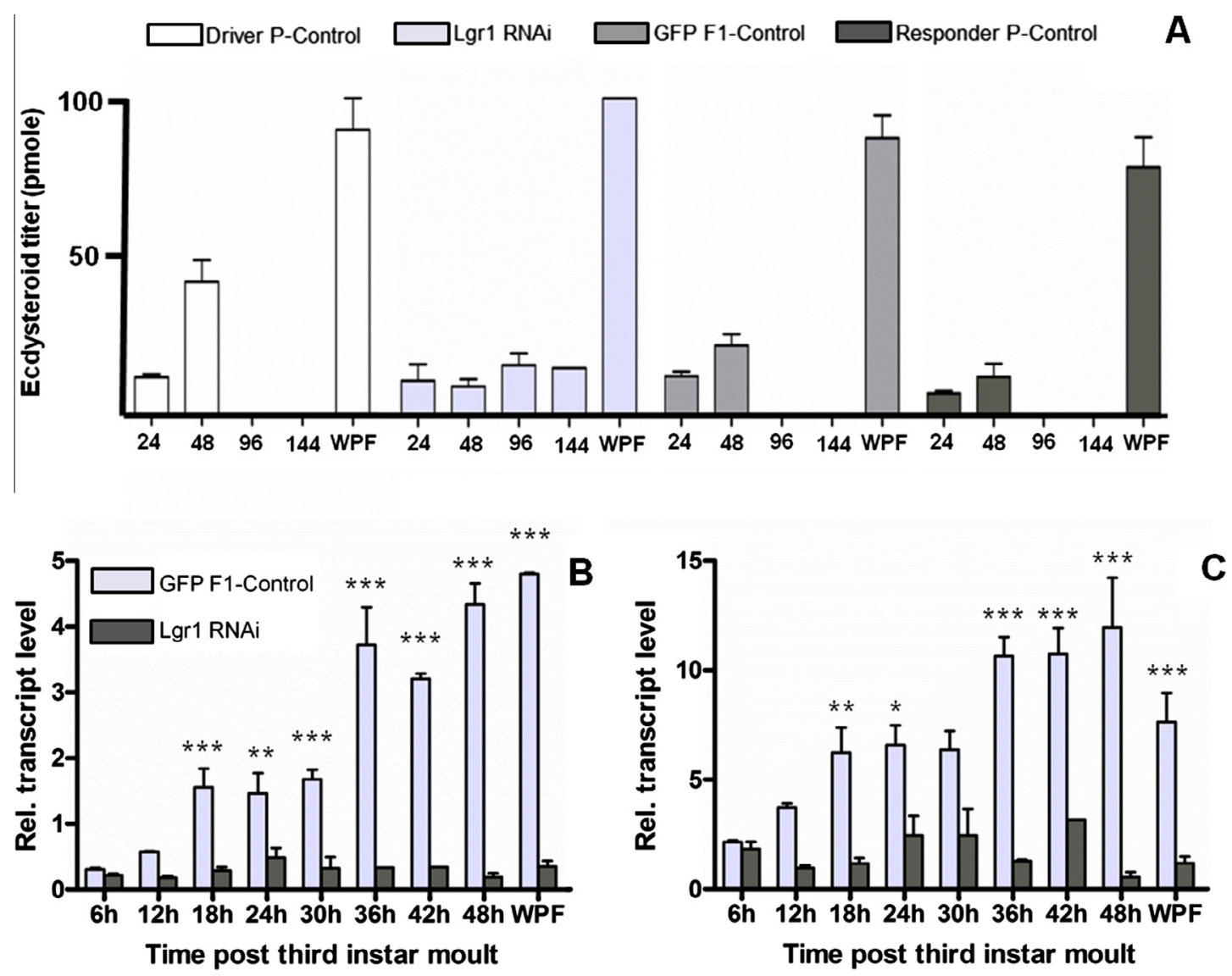

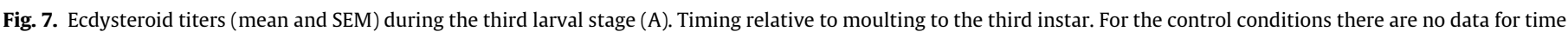

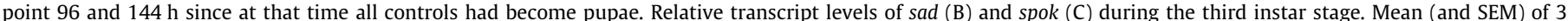
biological replicates. Significance of the difference was determined by Two way ANOVA with Bonferroni post hoc test $\left({ }^{* *} p<0.01\right.$, $\left.{ }^{* * *} p<0.001\right)$.

the salivary glands of third instar larvae, which is in accordance with high-throughput expression data [modENCODE (accessible via Flybase) and Chintapalli et al. (2007)]. This is also in line with previously described effects on the defecation rate of the glycoprotein hormone alpha subunit in C. elegans (Oishi et al., 2009). The modENCODE data set also reveals a significant transcript presence in the imaginal discs. These tissues were not included in our study, except for the gonadal discs, in which lgr1 transcripts were detected in the male but not the female. This discrepancy may point to a sex-specific role in gonadal development, but may also refer to a sex difference in the rate of development of the gonads. For example, it has been established that the male gonads grow faster than the female gonads (Kerkis, 1931).

Another striking sexual dimorphism was observed in the adult salivary glands with males displaying a significantly higher $\lg 1$ transcript abundance compared to females. In this case, we can remark that the FlyAtlas data (Chintapalli et al., 2007) indeed showed a high signal for the adult salivary glands but, since these samples were of mixed sexes, this high signal was likely to be attributed mainly to the transcript abundance in the male salivary glands. The approximately sixfold higher level of lgr1 transcript in the salivary glands of males compared to females may contribute greatly to the difference in lgr1 mRNA levels already observed by Hauser et al. (1997).

In contrast to the third instar, the CNS from adult flies displayed a notable $\lg 1$ transcript level which may indicate that a central role for LGR1 is more pronounced in the adult than in the third instar stage.
We demonstrated that transcripts for gpa2 and gpb5 were mainly found in the CNS, which in the adult consists of the brain and the thoracic-abdominal ganglion. Data from in situ hybridisations and a gpb5 reporter fly line support our results, since they indicated expression of gpa 2 and gpb5 in paired neurosecretory cells in the ventral nerve cord of fruit fly larvae and of gpb5 in the adult thoracic-abdominal ganglion and suboesophageal ganglion (Sellami et al., 2011). Therefore, it is possible that the GPA2/GPB5 heterodimer is a neurological signal that directs the function of fluid transporting epithelia, as was previously suggested by these authors.

In addition to the CNS, the adult salivary gland displayed a high relative gpa2 and gpb5 mRNA quantity but only in males. This is a potentially interesting observation, since it corresponds with the transcript profile for lgr1 and might suggest a paracrine role for the GPA2/GPB5 dimer in this organ. Similarly, in the mammalian ovary, a paracrine function was already described for the homologue of this hormone, thyrostimulin (Sun et al., 2010).

Interestingly, the patterns in transcript level for gpa2 and gpb5 were not always consistent which might indicate that at least some of the biological functions of GPA2 and GPB5 in the fruit fly may not require both proteins to form a heterodimer. The same conclusion was reached for the lancelet after a study of the homologues of these genes in this organism (Dos Santos et al., 2009). Additionally, for the related glycoprotein hormones, there have been reports on physiological roles of the free $\alpha$-subunit (Nemansky et al., 1998) and of the hCG $\beta$-subunit (Cole, 2009, 2010). In D. melanogaster, the bursicon $\alpha$ - and $\beta$-subunits, which are structurally related to 

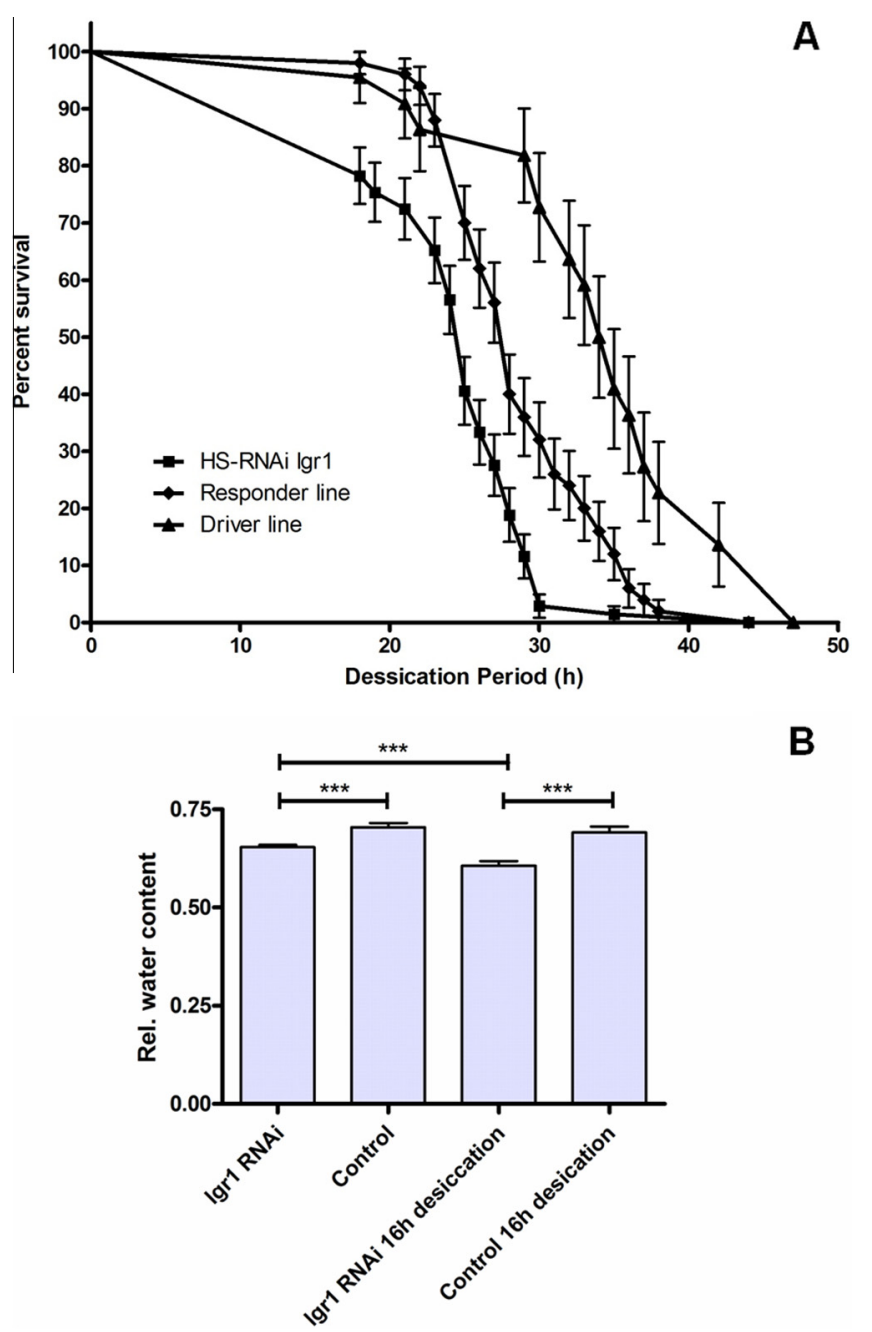

B

Fig. 8. Survival curves of adult males under desiccating circumstances (A). Lgr1 knockdown males succumb to desiccation significantly sooner than the control males. Survival curves were compared using the log-rank test $(n=70-90$, $p<0.0001)$. Driver line: Hsp70> Gal4; responder line: UAS-lgr1 RNAi; HS-RNAi lgr1: offspring of the (driver line $\times$ responder line) cross - RNAi in all tissues occurs after heat shock treatment. All conditions underwent identical heat shock treatment. Water content of adult flies (mean and SEM) normalised to the wet mass of the flies (B). Statistical significance was calculated using t-test $\left({ }^{* * *} p<0.001\right)$.

GPA2 and GPB5, were recently reported to form homodimers that can induce innate immunity genes (An et al., 2012).

\subsection{Lgr1 silencing phenotype}

We demonstrated that silencing lgr1 induced severe developmental defects with fruit fly larvae being impaired in their WPF. While our results were obtained by using only a single RNAi line, the sequence was carefully checked using bioinformatic tools to minimise the chances of off-target effects. Other RNAi lines targeting $\lg 1$ were tested but resulted in a much less pronounced transcript knockdown effect. Therefore, it is not surprising that they did not elicit the same potent phenotype. Most interestingly, our observations are remarkably well in line with the generation of significantly reduced numbers of adult flies when neurons producing LGR1-ligand (GPA2/GPB5) were genetically ablated (Sellami et al., 2011). As more adult flies still emerged in the latter study, the effects from ablation of the ligand-producing cells seemed less severe than those of $\lg 1$ silencing. An explanation for this might be that the receptor could show in vivo constitutive activity, as has been described for LGR1 expressed in a mammalian cell line (Nishi et al., 2000). This might have resulted in a basal level of receptor activity that was still sufficient to allow a substantial fraction of the flies to continue their development. In further support of our findings, in Tribolium castaneum, RNAi targeting of the beetle LGR1 homologue resulted in 50\% mortality in larval and pupal stages (Bai et al., 2011).

Given their rigid exoskeleton, the growth of insects depends on several moulting events during their lifetime. A fruit fly progresses through three larval stages and a pupal stage during which it undergoes metamorphosis to eventually emerge as an adult fly. During this developmental process, a strictly co-ordinated control of different organs and organ systems is required. Since untimely progression of a moult would have detrimental effects on the organism, each step is rigorously controlled by a cascade of hormones. Over time, significant research efforts have been devoted to the study of metamorphosis in D. melanogaster. A crucial player in this process is ecdysone, a steroid hormone from the prothoracic gland (PG) (in the case of D. melanogaster, incorporated in the ring gland), that is converted into $20 \mathrm{E}$ in target tissues. The latter is the active compound that induces the progression of a larva into the next developmental stage. Our data indeed show that silencing lgr1 significantly affected the ecdysteroid levels in third instar larvae. Lgr1 RNAi larvae had low ecdysteroid titers throughout their prolonged larval stage. In contrast, control larvae did display a peaking ecdysteroid titer associated with WPF. Only in the small fraction of pupa-forming lgr1 RNAi larvae, there was a rise in the ecdysteroid titer. Moreover, lgr1 RNAi larvae could be partially rescued by addition of ecdysteroid in their food. This suggests that the witnessed developmental defects may (in part) be associated with a derailing of the regulation of ecdysteroid biosynthesis.

The synthesis of ecdysone depends on the activity of cytochrome P450 enzymes encoded by the Halloween genes (Warren et al., 2004; Marchal et al., 2011), but a number of steps in this biosynthesis pathway remain unidentified and are represented by the 'black box' (Ono et al., 2012; Warren et al., 2009). To gain more insight into where in the pathway LGR1 signalling may play a role, we studied the transcript levels of a number of these genes. We found that transcript levels for the Halloween genes sad and spok were significantly decreased in wandering third instar larvae as a consequence of $\lg 1$ silencing. Sad encodes a 2-hydroxylase which is required to convert 2-deoxyecdysone into ecdysone (Warren et al., 2002). This latter hormone is hydroxylated at position 20 to form the more active compound 20-hydroxyecdysone, 20E, a reaction catalysed by Shade (shd) (Petryk et al., 2003). The precise role of the gene product of spok is unknown, but it probably catalyses the rate limiting reaction in the black box (Ono et al., 2012). Likely, the lowered transcript abundance of sad and spok are directly responsible for the absence of ecdysteroid build-up in the majority of third instar lgr1 RNAi larvae. The larvae that were able to undergo WPF displayed ecdysteroid levels comparable to the control conditions, but these larvae did require more time to raise these hormone levels. Of course, this population can reasonably be regarded as biased for the well-off individuals, i.e. those in which the knockdown of lgr1 was less potent. Since the release of ecdysteroids is crucial to ensure a correct progression of metamorphosis and the processes leading up to it, its levels are tightly regulated. Under normal circumstances, small peaks in the circulating titers occur at 8,20 and $28 \mathrm{~h}$ after third instar ecdysis. The first of these peaks has been associated with the attainment of critical weight. The latter two are linked to the switch in the fate of the salivary gland and the onset of wandering behaviour, respectively. Following these small peaks, a high titer of 20E signals the onset of WPF (Warren et al., 2006).

In addition to ecdysteroid levels, there are also other molecular indicators of the progression of the third instar stage. Whereas 
the genes sgs4 and ilp6 normally rise in transcript level in association with the wandering stage, in lgr1 RNAi larvae, sgs4 transcript levels remained nearly undetectable and ilp6 only displayed a minor rise in transcript abundance. This points to a profound deregulation of the processes preparing the larva for metamorphosis. Around the time of switching to the wandering behaviour, the salivary glands' production is altered, as can be observed from the rise in transcript presence of salivary gland secretion genes, such as sgs4, and the fall of transcript level of other secretion genes, such as new glue. This changes the fate of the salivary gland from a secretory organ in the digestive system to a production site of glue compounds that allow the larva to adhere to a suitable surface for pupariation (Biyasheva et al., 2001). The insulin-like peptide (ILP) encoding gene ilp6 was demonstrated to function as a growth factor in the absence of feeding. Indeed, during the wandering stage, tissue growth and development must be continued even though larvae do not feed during this period and therefore cannot rely on 'standard' insulin signalling (Okamoto et al., 2009; Slaidina et al., 2009). The absence of a strong increase in ilp6 transcript levels may be linked with the abnormally low ecdysteroid levels and with the incomplete wandering behaviour that we observed in the lgr1 RNAi larvae. Without ILP6 taking over insulin-like signalling and thus providing growth potential, the starvation during wandering may require the larva to reinitiate 'standard' insulin-like signalling. In order to do so, the larva will have to acquire nutrients, forcing it to return to the food source. Indeed, lgr1 RNAi larvae remained in or in close proximity of the food medium.

Linked with this subject, the gain of weight was lower in $\lg 1$ RNAi larvae in comparison to controls. Although this strongly suggests that the developmental threshold of reaching critical weight was not passed, this is unlikely the sole cause underlying the developmental defect. If that were the case, the result would be a delay in development rather than an arrest. This is supported by the observation that if a larva is starved before critical weight has been reached and is then returned to a food source, the larva will correctly undergo metamorphosis after having fed sufficiently (Mirth and Riddiford, 2007).

The regulatory network that is associated with moulting and metamorphosis is incredibly complex and includes long-described molecules such as JH, PTTH and 20E, but additional players in the pathway are still being identified today (Marchal et al., 2010; Tanaka, 2011; Zitnan, 2012). The receptor for PTTH has been characterised as Torso (Rewitz et al., 2009). But since PTTH deficiency only evokes a delay in development, not an arrest, several other factors are likely controlling ecdysteroid biosynthesis by the PG (McBrayer et al., 2007; Marchal et al., 2010). ILP signalling (IS) has been implicated in the control of ecdysone production, which in turn was demonstrated to affect growth rate (Mirth et al., 2005; Colombani et al., 2005; Caldwell et al., 2005). Recently, transforming growth factor $\beta$ (TGF $\beta$ )/activin signalling has also been shown to be required for torso expression and thus likely sensitises the prothoracic gland for the PTTH signal. Furthermore, a reduction of activin signalling reduces the levels of $D$. melanogaster insulin receptor, likely a nutritional feed into the regulation of $20 \mathrm{E}$ release (Gibbens et al., 2011). Furthermore, the newly described insulin-like peptide, Drosophila ILP8, was demonstrated to be released from the imaginal discs in case of tissue damage or tumourous growth and resulted in a developmental delay of the larva (Colombani et al., 2012; Garelli et al., 2012).

Our results indicate that this regulatory system may be even more complex, since the described pupariation defects resulting from $\lg 1$ silencing in fruit fly larvae point to an additional regulatory input. As demonstrated, knocking down lgr1 transcript in the PG did not result in developmental defects; therefore, LGR1 signalling likely controls pupariation peripherally. In this context, it is worth mentioning that several other data from literature already indicated that ecdysteroidogenesis may indeed be subjected to a remote control, such as MIP/PTSP produced in the epiproctodeal glands in Bombyx mori (Gelman and Beckage, 1995; Gelman and Bell, 1995; Yamanaka et al., 2010).

\subsection{LGR1 and a role in osmoregulation}

In support of the proposed role for LGR1 signalling in osmoregulation, our data using lgr 1 silenced adults indicated a significant reduction in survival under desiccating circumstances. Furthermore, the water content of these lgr1 RNAi flies was significantly lower than in controls and their loss of water during desiccation was significantly greater. Given the high transcript abundance of lgr1 in the hindgut of both third instar and adult fruit flies, the receptor's main role may lie in the control of an antidiuretic pathway, as previously proposed by Sellami et al. (2011) for the GPA2/ GPB5 hormone. More recently, GPA2/GPB5 was indeed shown to affect the transport of $\mathrm{Na}+$ and $\mathrm{K}+$ ions across the ileum and the rectum of the mosquito Aedes aegypti. Under the influence of the heterodimer the ileum's secretion of $\mathrm{Na}+$ decreased as did the absorption of $\mathrm{K}+$ in this tissue. In the rectum, GPA2/GPB5 increased the absorption of $\mathrm{Na}+$ but decreased the absorption of $\mathrm{K}+$. The authors concluded that GPA2/GPB5 functions as a regulator ionic balance, for example after the mosquito's blood meal (Paluzzi et al., 2014).

Given this role in the homeostasis of water and ions, it is plausible that intervening in LGR1 signalling may cause a deregulation that is significant enough to disrupt important physiological and developmental processes, such as the regulation of ecdysteroid biosynthesis and pupariation. In literature, several reports point at the existence of a possible link between the homeostasis of water and salts and developmental events in arthropods (Zdarek and Fraenkel, 1970; Chung et al., 1999; Cognigni et al., 2011; Van Wielendaele et al., 2012). Haemolymph volume and osmotic pressure are strongly regulated in relation to moulting and metamorphosis (Lee, 1961; Mills and Whitehead, 1970). Therefore, we hypothesise that a remote control system may exist in insects which coordinates both the developmental and the anti-diuretic physiological activities and that GPA2/GPB5-LGR1 signalling pathway plays a prominent role herein. The role of this pathway may be very ancient, since its components show conservation across animal phyla and also in chordates a homologous system (TSH-TSHR) appears to play a crucial role in regulating important lifecycle transitions, such as metamorphosis (Laudet, 2011). Moreover, our study may constitute an interesting basis for stimulating further research on this fascinating switch system in insects, as well as in many other groups of the animal kingdom.

\section{Conclusion}

In this paper, we have elucidated the developmental and tissue distribution of the transcripts for $\lg 1$ and for the genes encoding the glycoprotein hormone related subunits, gpa2 and gpb5. Furthermore, we have described the phenotype associated with $\lg 1$ transcript silencing. This included a prolonged third instar stage and a developmental arrest as third instar or early pupa. The reduction of the transcript levels of the Halloween genes sad and spok as well as of the genes sgs4 and ilp6 suggest that the observed defects were potentially a consequence of a decrease of ecdysteroid levels. Moreover, adults in which lgr1 had been silenced using a heat shock Gal4 driver died sooner under desiccating circumstances than control flies. 


\section{Acknowledgments}

The authors gratefully acknowledge M.B. O'Connor and L. Riddiford for the kindly donated ring gland-specific Gal4-driver lines and J.-P. Delbecque for the kindly provided antibody and tracer solutions for ecdysteroid quantification. They also thank J. Van Duppen for excellent technical assistance. Funding for this study was provided by the Interuniversity Attraction Poles program [Belgian Science Policy Grant (P7/40)], the Flemish (FWO) and the KU Leuven (GOA/11/02) Research Foundation. HPV was supported by a FLOF fellowship of the KU Leuven.

\section{Appendix A. Supplementary data}

Supplementary data associated with this article can be found, in the online version, at http://dx.doi.org/10.1016/j.ygcen.2014.0 8.006 .

\section{References}

Allan, C.M., Kalak, R., Dunstan, C.R., McTavish, K.J., Zhou, H., Handelsman, D.J., Seibel, M.J., 2010. Follicle-stimulating hormone increases bone mass in female mice. Proc. Natl. Acad. Sci. USA 107, 22629-22634.

An, S., Dong, S., Wang, Q., Li, S., Gilbert, L.I., Stanley, D., Song, Q., 2012. Insect neuropeptide bursicon homodimers induce innate immune and stress genes during molting by activating the NF-kappaB transcription factor Relish. PLoS One 7 , e34510.

Bai, H., Zhu, F., Shah, K., Palli, S.R., 2011. Large-scale RNAi screen of G proteincoupled receptors involved in larval growth, molting and metamorphosis in the red flour beetle. BMC Genomics 12, 388.

Biyasheva, A., Do, T.V., Lu, Y., Vaskova, M., Andres, A.J., 2001. Glue secretion in the Drosophila salivary gland: a model for steroid-regulated exocytosis. Dev. Biol. 231, 234-251.

Caldwell, P.E., Walkiewicz, M., Stern, M., 2005. Ras activity in the Drosophila prothoracic gland regulates body size and developmental rate via ecdysone release. Curr. Biol. 15, 1785-1795.

Chen, H., Ge, R.S., Zirkin, B.R., 2009. Leydig cells: from stem cells to aging. Mol. Cell. Endocrinol. 306, 9-16.

Chintapalli, V.R., Wang, J., Dow, J.A., 2007. Using FlyAtlas to identify better Drosophila melanogaster models of human disease. Nat. Genet. 39, 715-720.

Chung, J.S., Dircksen, H., Webster, S.G., 1999. A remarkable, precisely timed release of hyperglycemic hormone from endocrine cells in the gut is associated with ecdysis in the crab Carcinus maenas. Proc. Natl. Acad. Sci. USA 96, 13103-13107.

Cognigni, P., Bailey, A.P., Miguel-Aliaga, I., 2011. Enteric neurons and systemic signals couple nutritional and reproductive status with intestinal homeostasis. Cell Metab. 13, 92-104.

Cole, L.A., 2010. Biological functions of hCG and hCG-related molecules. Reprod. Biol. Endocrinol. 8, 102.

Cole, L.A., 2009. New discoveries on the biology and detection of human chorionic gonadotropin. Reprod. Biol. Endocrinol. 7, 8.

Colombani, J., Bianchini, L., Layalle, S., Pondeville, E., Dauphin-Villemant, C., Antoniewski, C., Carré, C., Noselli, S., Léopold, P., 2005. Antagonistic actions of ecdysone and insulins determine final size in Drosophila. Science 28 (310), 667670.

Colombani, J., Andersen, D.S., Leopold, P., 2012. Secreted peptide Dilp8 coordinates Drosophila tissue growth with developmental timing. Science 336, 582-585.

Dos Santos, S., Bardet, C., Bertrand, S., Escriva, H., Habert, D., Querat, B., 2009. Distinct expression patterns of glycoprotein hormone-alpha2 and -beta5 in a basal chordate suggest independent developmental functions. Endocrinology 150, 3815-3822.

Garelli, A., Gontijo, A.M., Miguela, V., Caparros, E., Dominguez, M., 2012. Imaginal discs secrete insulin-like peptide 8 to mediate plasticity of growth and maturation. Science 336, 579-582.

Gelman, D.B., Beckage, N.E., 1995. Low-molecular-weight ecdysiotropins in proctodaea of 5th instars of the tobacco hornworm, Manduca sexta (Lepidoptera, Sphingidae), and hosts parasitized by the braconid wasp, Cotesia congregata (Hymenoptera, Braconidae). Eur. J. Entomol. 92, 123-129.

Gelman, D.B., Bell, R.A., 1995. Low-molecular-weight ecdysiotropins in the hemolymph of 5 th instars of the European corn-borer, Ostrinia nubilalis (Lepidoptera, Pyralidae), and the gypsy-moth, Lymantria dispar (Lepidoptera, Lymantriidae). Eur. J. Entomol. 92, 131-141.

Gibbens, Y.Y., Warren, J.T., Gilbert, L.I., O'Connor, M.B., 2011. Neuroendocrine regulation of Drosophila metamorphosis requires TGFbeta/Activin signaling. Development 138, 2693-2703.

Gogakos, A.I., Duncan Bassett, J.H., Williams, G.R., 2010. Thyroid and bone. Arch. Biochem. Biophys. 503, 129-136.

Graveley, B.R., Brooks, A.N., Carlson, J.W., Duff, M.O., Landolin, J.M., Yang, L., Artieri, C.G., van Baren, M.J., Boley, N., Booth, B.W., Brown, J.B., Cherbas, L., Davis, C.A., Dobin, A., Li, R., Lin, W., Malone, J.H., Mattiuzzo, N.R., Miller, D., Sturgill, D., Tuch, B.B., Zaleski, C., Zhang, D., Blanchette, M., Dudoit, S., Eads, B., Green, R.E.,
Hammonds, A., Jiang, L., Kapranov, P., Langton, L., Perrimon, N., Sandler, J.E. Wan, K.H., Willingham, A., Zhang, Y., Zou, Y., Andrews, J., Bickel, P.J., Brenner, S.E., Brent, M.R., Cherbas, P., Gingeras, T.R., Hoskins, R.A., Kaufman, T.C., Oliver, B., Celniker, S.E., 2011. The developmental transcriptome of Drosophila melanogaster. Nature 471, 473-479.

Hauser, F., Nothacker, H.P., Grimmelikhuijzen, C.J., 1997. Molecular cloning, genomic organization, and developmental regulation of a novel receptor from Drosophila melanogaster structurally related to members of the thyroidstimulating hormone, follicle-stimulating hormone, luteinizing hormone/ choriogonadotropin receptor family from mammals. J. Biol. Chem. 272, 10021010.

Jiang, X., Liu, H., Chen, X., Chen, P.H., Fischer, D., Sriraman, V., Yu, H.N., Arkinstall, S. He, X., 2012. Structure of follicle-stimulating hormone in complex with the entire ectodomain of its receptor. Proc. Natl. Acad. Sci. USA 109, 12491-12496.

Kajava, A.V., Vassart, G., Wodak, S.J., 1995. Modeling of the three-dimensional structure of proteins with the typical leucine-rich repeats. Structure 3, 867877.

Katoh, K., Kuma, K., Toh, H., Miyata, T., 2005. MAFFT version 5: improvement in accuracy of multiple sequence alignment. Nucleic Acids Res. 33, 511-518.

Katoh, K., Misawa, K., Kuma, K., Miyata, T., 2002. MAFFT: a novel method for rapid multiple sequence alignment based on fast Fourier transform. Nucleic Acids Res. 30, 3059-3066.

Kerkis, J., 1931. The growth of the gonads in Drosophila melanogaster. Genetics 16, 212-224.

Kimura, K., Kodama, A., Hayasaka, Y., Ohta, T., 2004. Activation of the cAMP/PKA signaling pathway is required for post-ecdysial cell death in wing epidermal cells of Drosophila melanogaster. Development 131, 1597-1606.

Laan, M., Grigorova, M., Huhtaniemi, I.T., 2012. Pharmacogenetics of folliclestimulating hormone action. Curr. Opin. Endocrinol. Diabetes Obes. 19, 220-227.

Laudet, V., 2011. The origins and evolution of vertebrate metamorphosis. Curr. Biol. 21 (18), R726-737.

Lee, R.M., 1961. The variation of blood volume with age in the desert locust (Schistocerca gregaria Forsk.). J. Insect Physiol. 6, 36-51.

Loveall, B.J., Deitcher, D.L., 2010. The essential role of bursicon during Drosophila development. BMC Dev. Biol. 10, 92.

Luo, C.W., Dewey, E.M., Sudo, S., Ewer, J., Hsu, S.Y., Honegger, H.W., Hsueh, A.J., 2005 Bursicon, the insect cuticle-hardening hormone, is a heterodimeric cystine knot protein that activates $G$ protein-coupled receptor LGR2. Proc. Natl. Acad. Sci. USA 102, 2820-2825.

Marchal, E., Vandersmissen, H.P., Badisco, L., Van de Velde, S., Verlinden, H., Iga, M., Van Wielendaele, P., Huybrechts, R., Simonet, G., Smagghe, G., Vanden Broeck, J., 2010. Control of ecdysteroidogenesis in prothoracic glands of insects: a review Peptides 31, 506-519.

Marchal, E., Badisco, L., Verlinden, H., Vandersmissen, T., Van Soest, S., Van Wielendaele, P., Vanden Broeck, J., 2011. Role of the halloween genes, spook and phantom in ecdysteroidogenesis in the desert locust, Schistocerca gregaria. J. Insect Physiol. 57, 1240-1248.

McBrayer, Z., Ono, H., Shimell, M., Parvy, J.P., Beckstead, R.B., Warren, J.T., Thummel C.S., Dauphin-Villemant, C., Gilbert, L.I., O'Connor, M.B., 2007 Prothoracicotropic hormone regulates developmental timing and body size in Drosophila. Dev. Cell 13, 857-871.

Mendive, F.M., Van Loy, T., Claeysen, S., Poels, J., Williamson, M., Hauser, F. Grimmelikhuijzen, C.J., Vassart, G., Vanden Broeck, J., 2005. Drosophila molting neurohormone bursicon is a heterodimer and the natural agonist of the orphan receptor DLGR2. FEBS Lett. 579, 2171-2176.

Mills, R.R., Whitehead, D.L., 1970. Hormonal control of tanning in the American cockroach: changes in blood cell permeability during ecdysis. J. Insect Physiol. $16,331-340$.

Mirth, C.K., Truman, J.W., Riddiford, L.M., 2005. The role of the prothoracic gland in determining critical weight for metamorphosis in Drosophila melanogaster. Curr. Biol. 15, 1796-1807.

Mirth, C.K., Riddiford, L.M., 2007. Size assessment and growth control: how adult size is determined in insects. BioEssays 29, 344-355.

Nakabayashi, K., Matsumi, H., Bhalla, A., Bae, J., Mosselman, S., Hsu, S.Y., Hsueh, A.J., 2002. Thyrostimulin, a heterodimer of two new human glycoprotein hormone subunits, activates the thyroid-stimulating hormone receptor. J. Clin. Invest. 109, 1445-1452.

Natzle, J.E., Kiger Jr., J.A., Green, M.M., 2008. Bursicon signaling mutations separate the epithelial-mesenchymal transition from programmed cell death during Drosophila melanogaster wing maturation. Genetics 180, 885-893.

Nemansky, M., Moy, E., Lyons, C.D., Yu, I., Blithe, D.L., 1998. Human endometrial stromal cells generate uncombined alpha-subunit from human chorionic gonadotropin, which can synergize with progesterone to induce decidualization. J. Clin. Endocrinol. Metab. 83, 575-581.

Nishi, S., Hsu, S.Y., Zell, K., Hsueh, A.J., 2000. Characterization of two fly LGR (leucine-rich repeat-containing, G protein-coupled receptor) proteins homologous to vertebrate glycoprotein hormone receptors: constitutive activation of wild-type fly LGR1 but not LGR2 in transfected mammalian cells. Endocrinology 141, 4081-4090.

Oishi, A., Gengyo-Ando, K., Mitani, S., Mohri-Shiomi, A., Kimura, K.D., Ishihara, T., Katsura, I., 2009. FLR-2, the glycoprotein hormone alpha subunit, is involved in the neural control of intestinal functions in Caenorhabditis elegans. Genes Cells $14,1141-1154$

Okamoto, N., Yamanaka, N., Yagi, Y., Nishida, Y., Kataoka, H., O'Connor, M.B., Mizoguchi, A., 2009. A fat body-derived IGF-like peptide regulates postfeeding growth in Drosophila. Dev. Cell 17, 885-891. 
Ono, H., Rewitz, K.F., Shinoda, T., Itoyama, K., Petryk, A., Rybczynski, R., Jarcho, M., Warren, J.T., Marques, G., Shimell, M.J., Gilbert, L.I., O'Connor, M.B., 2006. Spook and spookier code for stage-specific components of the ecdysone biosynthetic pathway in Diptera. Dev. Biol. 298, 555-570.

Ono, H., Morita, S., Asakura, I., Nishida, R., 2012. Conversion of 3-oxo steroids into ecdysteroids triggers molting and expression of 20E-inducible genes in Drosophila melanogaster. Biochem. Biophys. Res. Commun. 421, 561-566.

Paluzzi, J.-P., Vanderveken, M., O'Donnell, M.J., 2014. The heterodimeric glycoprotein hormone, GPA2/GPB5, regulates ion transport across the hindgut of the adult mosquito, Aedes aegypti. PLoS One 9, e86386.

Peabody, N.C., Diao, F., Luan, H., Wang, H., Dewey, E.M., Honegger, H.W., White, B.H., 2008. Bursicon functions within the Drosophila CNS to modulate wing expansion behavior, hormone secretion, and cell death. J. Neurosci. 28 14379-14391.

Petryk, A., Warren, J.T., Marques, G., Jarcho, M.P., Gilbert, L.I., Kahler, J., Parvy, J.P., Li, Y., Dauphin-Villemant, C., O'Connor, M.B., 2003. Shade is the Drosophila P450 enzyme that mediates the hydroxylation of ecdysone to the steroid insect molting hormone 20-hydroxyecdysone. Proc. Natl. Acad. Sci. USA 100, 13773 13778 .

Radu, A., Pichon, C., Camparo, P., Antoine, M., Allory, Y., Couvelard, A., Fromont, G., Hai, M.T., Ghinea, N., 2010. Expression of follicle-stimulating hormone receptor in tumor blood vessels. N. Engl. J. Med. 363, 1621-1630.

Rewitz, K.F., Yamanaka, N., Gilbert, L.I., O'Connor, M.B., 2009. The insect neuropeptide PTTH activates receptor tyrosine kinase torso to initiate metamorphosis. Science 326, 1403-1405.

Sellami, A., Agricola, H.J., Veenstra, J.A., 2011. Neuroendocrine cells in Drosophila melanogaster producing GPA2/GPB5, a hormone with homology to LH, FSH and TSH. Gen. Comp. Endocrinol. 170, 582-588.

Slaidina, M., Delanoue, R., Gronke, S., Partridge, L., Leopold, P., 2009. A Drosophila insulin-like peptide promotes growth during nonfeeding states. Dev. Cell 17, 874-884.

Smits, G., Campillo, M., Govaerts, C., Janssens, V., Richter, C., Vassart, G., Pardo, L., Costagliola, S., 2003. Glycoprotein hormone receptors: determinants in leucinerich repeats responsible for ligand specificity. EMBO J. 22, 2692-2703.

Sudo, S., Kuwabara, Y., Park, J.I., Hsu, S.Y., Hsueh, A.J., 2005. Heterodimeric fly glycoprotein hormone-alpha2 (GPA2) and glycoprotein hormone-beta5 (GPB5) activate fly leucine-rich repeat-containing $G$ protein-coupled receptor-1 (DLGR1) and stimulation of human thyrotropin receptors by chimeric fly GPA2 and human GPB5. Endocrinology 146, 3596-3604.

Sun, L., Peng, Y., Sharrow, A.C., Iqbal, J., Zhang, Z., Papachristou, D.J., Zaidi, S., Zhu, L.L., Yaroslavskiy, B.B., Zhou, H., Zallone, A., Sairam, M.R., Kumar, T.R., Bo, W. Braun, J., Cardoso-Landa, L., Schaffler, M.B., Moonga, B.S., Blair, H.C., Zaidi, M. 2006. FSH directly regulates bone mass. Cell 125, 247-260.
Sun, S.C., Hsu, P.J., Wu, F.J., Li, S.H., Lu, C.H., Luo, C.W., 2010. Thyrostimulin, but not thyroid-stimulating hormone (TSH), acts as a paracrine regulator to activate the TSH receptor in mammalian ovary. J. Biol. Chem. 285, 3758-3765.

Tamura, K., Peterson, D., Peterson, N., Stecher, G., Nei, M., Kumar, S., 2011. MEGA5: molecular evolutionary genetics analysis using maximum likelihood, evolutionary distance, and maximum parsimony methods. Mol. Biol. Evol. 28, 2731-2739.

Tanaka, Y., 2011. Recent topics on the regulatory mechanism of ecdysteroidogenesis by the prothoracic glands in insects. Front. Endocrinol. (Lausanne) 2, 107.

Van Hiel, M.B., Vandersmissen, H.P., Van Loy, T., Vanden Broeck, J., 2012. An evolutionary comparison of leucine-rich repeat containing G protein-coupled receptors reveals a novel LGR subtype. Peptides 34, 193-200.

Van Wielendaele, P., Dillen, S., Marchal, E., Badisco, L., Vanden Broeck, J., 2012. CRFlike diuretic hormone negatively affects both feeding and reproduction in the desert locust, Schistocerca gregaria. PLoS One 7, e31425.

Vandesompele, J., De Preter, K., Pattyn, F., Poppe, B., Van Roy, N., De Paepe, A. Peleman, F., 2002. Accurate normalization of real-time quantitative RT-PCR data by geometric averaging of multiple internal control genes. Genome Biol. 3.

Warren, J.T. O'Connor, M.B., Gilbert, L.I., 2009. Studies on the Black Box: incorporation of 3-oxo-7-dehydrocholesterol into ecdysteroids by Drosophila melanogaster and Manduca sexta. Insect Biochem. Mol. Biol. 39, 677-687.

Warren, J.T., Petryk, A., Marques, G., Jarcho, M., Parvy, J.P., Dauphin-Villemant, C., O'Connor, M.B., Gilbert, L.I., 2002. Molecular and biochemical characterization of two P450 enzymes in the ecdysteroidogenic pathway of Drosophila melanogaster. Proc. Natl. Acad. Sci. USA 99, 11043-11048.

Warren, J.T., Petryk, A., Marques, G., Parvy, J.P., Shinoda, T., Itoyama, K., Kobayashi, J., Jarcho, M., Li, Y., O'Connor, M.B., Dauphin-Villemant, C., Gilbert, L.I., 2004. Phantom encodes the 25-hydroxylase of Drosophila melanogaster and Bombyx mori: a P450 enzyme critical in ecdysone biosynthesis. Insect Biochem. Mol. Biol. 34, 991-1010.

Warren, J.T., Yerushalmi, Y., Shimell, M.J., O'Connor, M.B., Restifo, L.L., Gilbert, L.I., 2006. Discrete pulses of molting hormone, 20-hydroxyecdysone, during late larval development of Drosophila melanogaster: correlations with changes in gene activity. Dev. Dyn. 235, 315-326.

Yamanaka, N., Hua, Y.J., Roller, L., Spalovská-Valachová, I., Mizoguchi, A., Kataoka, H., Tanaka, Y., 2010. Bombyx prothoracicostatic peptides activate the sex peptide receptor to regulate ecdysteroid biosynthesis. Proc. Natl. Acad. Sci. USA 107 (5), 2060-2065.

Zdarek, J., Fraenkel, G., 1970. Overt and covert effects of endogenous and exogenous ecdysone in puparium formation of flies. Proc. Natl. Acad. Sci. USA 67, 331-337.

Zitnan, D., 2012. Neuroendocrine regulation of ecdysis. In: Gilbert, L.I. (Ed.), Insect Endocrinology. Academic Press (Elsevier), pp. 253-309, isbn: 978-0-12384749-2. 